\title{
XXXVII.-Silver Amalgams.
}

\section{By Henry Chapman Jones.}

Silver amalgams are of interest, not only because they are members of the general class of amalgams, but also because some occur in Nature, and because of the specific gravity of some of them being much higher than that of mercury. The remarkable contraction that takes place when the two metals combine was stated by Joule (Journ. Chem. Soc., 1863, 16, 383) to be "referable no doubt to the assumption of the solid state by the mercury, the specific gravity of which comes out at 16.5 from these (Joule's) experiments." This high value for the specific gravity of solid mercury does not appear to have been confirmed.

The silver amalgams that have so far been examined have been of very irregular compositions when viewed as compounds, and as they have been prepared by bringing the two metals together either directly, or by allowing an excess of mercury to precipitate silver on itself from a salt solution, it is hardly possible that the amalgams obtained were homogeneous.

The object of this paper is to indicate a method by which a series of silver amalgams may be obtained of definite molecular composition (within the errors of experiment) and in a really homogeneous condition; and, further, to give some of the properties of two of them.

The method consists, in short, in the preparation of a double salt, or a molecular mixture of salts, of the two metals with subsequent reduction in the cold to the metallic condition. When mercuric chloride acts on either silver or mercury, or an amalgam of the two metals, each atom of either metal combines with a molecule of mercuric chloride. If, then, the chlorine is removed, and this is easily done by means of ferrous oxalate, the metals alone remain. In this way amalgams may be obtained in which the metals exist in the proportions indicated by the formulæ $\mathrm{AgHg}$, $\mathrm{AgHg}_{3}, \mathrm{AgHg}_{7}, \mathrm{AgHg}_{15}, \mathrm{AgHg}_{31}, \mathrm{AgHg}_{63}$, and the author has actually prepared these six amalgams, although not all of them in 
quantities suitable for examination. If the product of the action of mercuric chloride on metallic silver, that is, the double chloride, $\mathrm{AgHgCl}$, is treated with an excess of sodium sulphite solution, half the silver and three-fourths of the mercury will be dissolved, and there will remain an amalgam of the composition represented by the formula $\mathrm{Ag}_{2} \mathrm{Hg}$. This, by treatment alternately with mercuric chloride and ferrous oxalate, gives another series of amalgams intermediate between the members of the series given above, namely, $\mathrm{Ag}_{2} \mathrm{Hg}, \mathrm{AgHg}_{2}, \mathrm{AgHg}_{5}, \mathrm{AgHg}_{11}$, etc.

\section{Preparation of the Amalgams.}

Some details concerning this have already been published $(J$. Soc. Chem. Ind., 1893, 12, 983). A granular sample of metallic silver reacts most readily with mercuric chloride-such preparations as are obtained by the reduction of silver salts by means of zinc in acid solutions, or by dextrose in the presence of alkali hydroxide. But metallic silver so obtained I have never found to be pure. If dried at $100^{\circ}$, it invariably loses something on ignition, $0.12,0.74$ per cent., and so on in different cases, although the product was well washed with dilute nitric acid and ammonia. Silver obtained by reduction with ammonium sulphite, if properly washed and dried at $100^{\circ}$, loses nothing on ignition, but such silver is flaky and crystalline rather than granular, and reacts less readily than the other. It is preferable, in using ammonium sulphite, to heat the mixture quickly in small portions, and so obtain the metal more finely divided than by the usual slow process.

Metallic silver and mercuric chloride will interact in many different liquids, or in no liquid, by simply shaking them together and allowing them to remain. I have always used water, but, judging from the analogous reaction with mercuric bromide, it might be possible to find a medium that would expedite the change. Mercuric bromide reacts very slowly in water, the salt being very sparingly soluble; but in benzene, in which the salt is soluble to practically the same extent, the velocity of the reaction is very remarkably increased, whilst in acetone, which very freely dissolves the mercuric bromide, the change does not appear to be so rapid as in benzene. Light petroleum dissolves much less of the mercuric salt than water does, yet under otherwise the same conditions the change will appear complete in light petroleum in a time that in water has sufficed for little more than a superficial reaction.

The reduction of the chloride by means of ferrous oxalate takes place practically at once. The reagent is obtained by pouring one volume of a saturated solution of ferrous sulphate into six volumes of a saturated solution of potassium oxalate. The large excess of 
the potassium oxalate is to make sure of keeping the iron salts in solution. The action of mercuric chloride on an amalgam so prepared, for the purpose of adding more mercury to it, is far more rapid than the action on metallic silver, presumably because the amalgam is more finely divided. If a double (or mixed) chloride of silver and mercury contains metallic silver (an unattacked residue, for example), it will not give a constant weight at $100^{\circ}$, as metallic mercury is liberated by the silver and continuously volatilised.

The Amalgam AgHg.-In bulk, as dried over sulphuric acid without the aid of heat (in a steam-oven such an amalgam lost mercury at a rate equivalent to 0.47 per cent. per hour), this amalgam appears as a grey, non-adherent powder, which can be easily burnished into a metallic film. It consists of rounded particles, generally about $0.003 \mathrm{~mm}$. in diameter. Some are oval and up to $0.005 \mathrm{~mm}$. long, and others are as small as $0.002 \mathrm{~mm}$. As diffused in cedarwood oil for microscopic examination, the particles have a marked tendency to adhere in rows, often branched, $\mathrm{Y}$-shape, but the individual particles are quite distinct, and there is no appearance of a want of homogeneity. The silver mercurous chloride from which it was obtained was in similar rounded particles, but more varied in size, and on the whole rather smaller. These differences are probably accidental, and it seems almost certain that on the reduction of the chloride to the amalgam the separate particles retain their individuality. The specific gravity of the amalgam, taken in water at $20^{\circ}$ and compared with water at $20^{\circ}$, was 12.8055 . When pumping out the air, there appeared to be a continual evolution of gas, as if the amalgam decomposed the water. A second estimation with more stringent methods to remove air gave 12.8099. A determination in xylene showed that these figures are not low, and therefore that if the amalgam does act on the water the result is not sufficient to vitiate the determination. The calculated specific gravity, assuming no contraction when the two metals combine, is 12.29 . Evidently, therefore, in the amalgam obtained as described, there is contraction on combination, but not nearly so much as in the crystalline amalgam which Joule obtained by placing mercury in silver nitrate solution. Such a preparation, which had approximately the same composition, had a specific gravity of $14 \cdot 68$, and another sample, obtained by adding more mercury and squeezing out as much as possible by a high pressure, gave 13.44. Other observers have found specific gravities for silver amalgams up to about 14 .

The Amalgam $\mathrm{AgHg}_{3}$. -When the amalgam $\mathrm{AgHg}$ is acted on by mercuric chloride, the resulting chloride (empirically $\mathrm{AgHg}_{3} \mathrm{Cl}_{4}$ ) 
is not distinguishable when viewed in bulk from the double chloride $\mathrm{AgHgCl}_{2}$; it is a white, soft-looking powder. When microscopically examined, it is at once clear that the characteristic structure of the double chloride, $\mathrm{AgHgCl}_{2}$, which persists in the amalgam $\mathrm{AgHg}$, produced from it by reduction, has now gone-the particles are disintegrated. They are smaller, $0.001 \mathrm{~mm}$. in diameter or less, and irregular in shape. This chloride is easily reduced, and the dried amalgam, $\mathrm{AgHg}_{3}$, appears in bulk as an adherent powder. If allowed to fall from the side of the bottle, it retains the shape of the bottle, much as undried sea-sand does. If rubbed with the side of a knife it crunches, and the bright metallic scale so produced appears, on magnification, to have globules of mercury exuding from it. If pushed up into a fold of wash-leather with a toothbrush handle, a large proportion of clean mercury globules can be pressed through the leather. When stirred into cedarwood oil and examined microscopically, it appears as crumbling, coherent, homogeneous masses, not separable into individual grains. The masses have no appearance of liquidity, their outlines are irregular and angular, especially the smaller detached particles, and the points tend to be blunt rather than spiky.

It is not proposed to continue this investigation, but Dr. J. C. Philip hopes to examine the physical properties of some of these amalgams, and to study the influence of the medium on the velocity of the reaction between mercuric halides and metallic silver.

Royal College of Science, South Kensington. 